\title{
Concerns of the anesthesiologist: anesthetic induction in severe sepsis or septic shock patients
}

\author{
Seok Hwa Yoon \\ Department of Anesthesiology and Pain Medicine, Chungnam National University School of Medicine, Daejeon, Korea
}

Septic patients portray instable hemodynamic states because of hypotension or cardiomyopathy, caused by vasodilation, thus, impairing global tissue perfusion and oxygenation threatening functions of critical organs. Therefore, it has become the primary concern of anesthesiologists in conducting anesthesia (induction, maintenance, recovery, and postoperative care), especially in the induction of those who are prone to fall into hemodynamic crisis, due to hemodynamic instability. The anesthesiologist must have a precise anesthetic plan based on a thorough preanesthetic evaluation because many cases are emergent. Primary circulatory status of patients, including mental status, blood pressure, urine output, and skin perfusion, are necessary, as well as more active assessment methods on intravascular volume status and cardiovascular function. Because it is difficult to accurately evaluate the intravascular volume, only by central venous pressure (CVP) measurements, the additional use of transthoracic echocardiography is recommended for the evaluation of myocardial performance and hemodynamic state. In order to hemodynamically stabilize septic patients, adequate fluid resuscitation must be given before induction. Most anesthetic induction agents cause blood pressure decline, however, it may be useful to use drugs, such as ketamine or etomidate, which carry less cardiovascular instability effects than propofol, thiopental and midazolam. However, if blood pressure is unstable, despite these efforts, vasopressors and inotropic agents must be administered to maintain adequate perfusion of organs and cellular oxygen uptake. (Korean J Anesthesiol 2012; 63: 3-10)

Key Words: Anesthetic induction, Intravenous anesthetics, Sepsis, Septic shock.

Received: June 4, 2012. Accepted: June 20, 2012.

Corresponding author: Seok Hwa Yoon, M.D., Department of Anesthesiology and Pain Medicine, Chungnam National University School of Medicine, 33, Munhwa-ro, Jung-gu, Daejeon 301-721, Korea. Tel: 82-42-280-7840, Fax: 82-42-280-7968, E-mail: seohwy@cnu.ac.kr

(c) This is an open-access article distributed under the terms of the Creative Commons Attribution Non-Commercial License (http:// creativecommons.org/licenses/by-nc/3.0/), which permits unrestricted non-commercial use, distribution, and reproduction in any medium, provided the original work is properly cited. 


\section{Introduction}

Despite continuous development in antibiotics, mechanical ventilation and renal replacement therapy, and outstanding surgical techniques, which contribute to decreasing death, severe sepsis and septic shock still leads to high death rate [1].

Sepsis is defined as the clinical syndrome defined by the presence of both infection and a systemic inflammatory response. Severe sepsis is defined as sepsis complicated by organ dysfunction, while septic shock is defined as severe sepsis plus a state of acute circulatory failure, characterized by persistent arterial hypotension (defined as a systolic arterial pressure below $90 \mathrm{mmHg}$, a mean arterial pressure $<60 \mathrm{mmHg}$ or a reduction in systolic blood pressure of $>40 \mathrm{mmHg}$ from baseline) unexplained by other causes and regardless of the adequate volume resuscitation [2].

Severe sepsis or septic shock usually causes progressive damage to multiple organs. In general, surgery is postponed until the initial treatment is initiated, but sometimes, it cannot be delayed when surgery itself is necessary to eliminate its cause. For instance, removal of abscess or infected prosthetic device, infective endocarditis, and bowel perforation cannot be postponed. In regarding anesthetic management in patients with severe sepsis or septic shock, the anesthesiologists' initial concern is generally to maintain adequate tissue perfusion, during hypotension. Because most anesthetics depress the cardiovascular system, maintaining adequate blood pressure is extremely stressful during an anesthetic induction and maintenance. Secondly, these cases are mostly emergent, rather than elective surgeries. Therefore, apprehension of the surgical and anesthetic status of patients is insufficient due to a lack of information. Also, during emergent surgeries, anesthetic induction is carried out in short of manpower and equipment. Thirdly, proper initial treatment is delayed due to speculating a wrong diagnosis of sepsis, which is generally due to an infection or rapid progression of sepsis leading to severe sepsis or septic shock. Thus, surgery is carried out without any specific plans on excluding the cause. Fourthly, efforts to establish evidence based treatment guidelines, such as the Surviving Sepsis Campaign (SSC) Guideline, focus on early detection and treatment. Most treatments are given in the emergency room or at the ward. However, it is questionable whether these guidelines are appropriate for those undergoing anesthesia, especially if these guidelines are applied under changes of the patient's physiological changes during general anesthesia.

The following describes 1) available anesthetic induction agents, 2) methods in evaluating volume status and fluid management, and 3) use of vasopressors and inotropic drugs in hemodynamic stabilization and support.

\section{The Available Induction Agents in the Severe Sepsis or Septic Shock}

Anesthetic drugs generally decrease myocardium contractility, and act directly on the heart and vasculature inducing vasodilation. Therefore, anesthesia itself may worsen the condition of these patients, weighing a substantial amount of burden and danger to the anesthesiologist during induction. All induction agents have a dose-dependent depression of cardiac work. Thus, maintaining hemodynamic stability during anesthetic induction is important, as well as choosing an ideal anesthetic. The following agents used in anesthetic induction are listed below with pros and cons. With the information provided, choosing the ideal agent is up to the anesthesiologist.

\section{Propofol}

Propofol belongs to a group of alkyl-phenol that has hypnotic effects, and is one of the most commonly used drugs in the intensive care unit, both for induction and for longer-term sedation. This is due to its rapid onset and is easily titrable, with the prospect of rapid recovery for the patient. In several experimental studies, it is also reported to have anti-inflammatory effects. Propofol increases bone morphogenetic protein-7 expression, decreases inflammatory cytokines, and inhibits oxidating stress, protecting the kidneys from sepsis-induced acute kidney injury. Also, propofol has beneficial effects in free radicals, lipid peroxidation-mediated oxidative injury and subsequent organ dysfunction in the endotoxemic pig in vivo [3]. During endotoxemia, antioxidative effects of propofol scavenge free radicals, affecting non-enzymatic lipid peroxidation through inhibition of F2-isoprostane formation. Also, an increase in plasmacytokines, such as TNF- $\alpha$, interleukin-6, and interleukin-10 induced by endotoxin, reduction in base deficit, and increase in infiltration neutrophils in the air space or vessel walls of the lungs, are attenuated by propofol [4]. In studies on hamsters pretreated with propofol, diaphragmatic dysfunction induced by septic peritonitis was improved. These beneficial effects of propofol are partially thought to be due to the inhibition of lipid peroxidation of the diaphragm, induced by powerful oxidants [5]. In rats with abdominal sepsis, clinical concentrations of propofol and midazolam depressed neutrophils hydrogen peroxide production. However, propofol showed more depression than midazolam [6]. Hypercoagulopathy is a common clinical feature of sepsis. Endotoxemia induces hypercoagulopathy by shortening the thromboplastin and thrombin times. In endotoxemic rats, administration of propofol decreased release of lipopolysaccharide (LPS)-stimulated serum platelet factor 4, and partially corrected hypercoagulopathy [7]. In studies using 
rats, the mortality rate of 5 hours after endotoxin injection was $73 \%$ for the endotoxic, $9 \%$ for the early posttreatment, and $36 \%$ for the late posttreatment groups with the additional administration of propofol. The early posttreatment of propofol after endotoxin injection drastically reduced the mortality rate of rats and attenuated their cytokine responses. Moreover, propofol attenuated the production of TNF- $\alpha$ [8]. Because of its anti-inflammatory effect, propofol is recommended as a sedative for septic patients in the ICU. However, in several experimental and clinical studies, propofol has proved to have the most pronounced adverse hemodynamic effects. Clinically, propofol suppresses the sympathetic vasoconstrictor activity, thus, decreasing systemic vascular resistance, cardiac contractility, and preload, Thereby, decreasing arterial blood pressure. Reich et al. [9] reported predictors of hypotension after anesthetic induction, which included: American Society of Anesthesiologists physical status classification III-V, baseline MAP $70 \mathrm{mmHg}$, age $50 \mathrm{yr}$, the use of propofol for induction of anesthesia, and increasing induction dosage of fentanyl. As a retrospective review, they recommended that it is advisable to avoid propofol induction in patients who present a baseline of MAP $70 \mathrm{mmHg}$. In a study using septic sheep, propofol deteriorated hemodynamics, such as renal blood flow declining $60 \%$ from the preseptic baseline and $39 \%$ from the septic value [10]. According to Zausig et al. [11], with propofol, cardiac contractility is decreased to $38 \%$, while lusitropy decreases $44 \%$, thus, having the most pronounced adverse direct cardiac effects. Some studies state that propofol lacks anti-inflammatory effect, and that it rather worsens the inflammatory responses to endotoxemia. Propofol increase TNF- $\alpha$ responses caused by LPS-stimulated human blood in vitro [12], and stimulates the production of TNF- $\alpha$, interleukin-1, and interleukin- 6 in critically ill surgical patients [13]. Therefore, whether or not propofol have anti-inflammatory effects in endotoxemia still remains unclear. This shows that propofol is not suitable for severe sepsis or septic shock patients, and it may in fact be harmful.

\section{Ketamine}

Ketamine is a phencyclidine derivative with characterizes in hypnosis, analgesia, and amnesia, and is frequently described as a unique drug. In contrast to other anesthetic agents, ketamine acts as a sympathomimetic to increase the heart rate, arterial blood pressure, and cardiac output. Ketamine has been recommended for anesthesia and sedation of septic or severely ill patients because of its cardiovascular stimulation effects. The inotropic support (dopamine, dobutamine, norepinephrine) could be reduced. In animal models of septic shock, ketamine preserves cardiovascular function well [14]. Also, in the isolated heart, ketamine increases the coronary perfusion and coronary oxygen supply. Although the increase in heart rate and contractility increase oxygen demand, the coronary reserve is not restricted [15]. Zausig et al [11]. reported that in the heart of septic rats, maximal cardiac work dysfunction occurred in the order of $\mathrm{s}(+)$-ketamine $(-6 \%)<$ etomidate $(-17 \%)<$ midazolam $(-38 \%)<\operatorname{propofol}(-50 \%)$.

There is a growing body of experimental evidence suggesting that ketamine exerts a protective anti-inflammatory effect against the sepsis process itself. Ketamine attenuates the production and release of cytokines in endotoxemia in vitro [16,17]. It is also reported that ketamine suppresses the induction of NO synthase activity and protein expression by endotoxin $[4,18]$. Administration of ketamine inhibited hypotension, metabolic acidosis, and cytokine responses in rats receiving a single intravenous bolus dose of endotoxin. An inhibitory effect was found even when initiation of treatment was delayed until 2 hours after the endotoxin exposure [19]. During sepsis and endotoxemia, hypoxic pulmonary vasoconstriction is damaged. Recently, Busch et al. [20] reported that ketamine modified the baseline pulmonary vascular properties, and as a result, decreased the hypoxic pulmonary vasoconstriction responsiveness in untreated mice.

Thus, the risks and benefits of ketamine must be considered thoroughly before use. Ketamine possesses undesirable psychotomimetic effects, such as illusion, disturbing dreams and delirium during emergence and recovery. Ketamine, although in the spectra of emergence delirium, may negate beneficial cardiovascular profile and protective effects against septic shock, in combination with midazolam or propofol may provide sedation and pain relief in ICU patients, which is extremely useful and safe, especially in septic patients with cardiovascular instability. However, ketamine has been contra-indicated in patients with increased intracranial pressure, but any adverse effects on intracranial pressure or cerebral blood flow are attenuated or reversed by controlled ventilation [14]. These results suggest that the proper use of ketamine, as an anesthetic agent, may offer certain advantages in the management of septic patients.

\section{Etomidate}

Etomidate, a popular choice, is an agent for the induction of unstable hemodynamic patients [21]. Etomidate, which is rapid and carries a predictable onset of action and recovery, is relatively hemodynamically stable, creates limited suppression of ventilation, lacks histamine release, and contains a favorably safe profile, and thus, effectively used in critical care settings. The standard induction dose of etomidate $(0.2-0.3 \mathrm{mg} / \mathrm{kg})$ creates rapid hypnosis within 5-15 seconds; hence, it is very 
predictable. This is the time from injection to when it reaches the brain. Patients injected with etomidate regained consciousness 5-14 minutes after injection [22].

However, the potential for adrenocortical suppression is an important consideration. 'CORTICUS' study confirmed that steroid suppression occurred $60 \%$ of septic patients who received etomidate, compared with $43 \%$ who did not, which is an effect that can continue up to $67 \mathrm{~h}$, indicating that the adverse effects are possibly important even in a single bolus dose [23]. Annane et al. [24] in the study of adrenal suppression, using etomidate, showed signs of adrenal insufficiency between 12 to 24 hours after injection in 68 patients who did not respond to the high-dose cosyntropin stimulation test, out of the 72 who were injected with etomidate during induction. This is congruent to other published reports. The mortality rate of the corticosteroid-treated group and the placebo-treated group were $75.7 \%$ and $54.8 \%$, respectively. Also, the incidence and mortality of adrenal insufficiency was investigated in a retrospective study of 65 patients with severe sepsis or in septic shock when inducted with etomidate (mean dose $0.3 \mathrm{mg} / \mathrm{kg}$ ) or midazolam [25]. Corticosteroids decrease the duration of septic shock, and is known to decrease the overall mortality. The biggest problem in using etomidate during induction is the adrenal suppression. However, injecting corticosteroids can completely reverse this problem. Murray and Marik [26] suggested that all hypotensive septic patients be treated with stress doses of corticosteroids, particularly, if using a random (stress) cortisol. Also, until the results of the stress cortisol level measurements are available, beginning administration of 100 mg of hydrocortisone intravenously is recommended, every 8 hours.

Arbous et al. [27] claims that $2 / 3$ of mortality during induction is due to cardiovascular events. The clinical significance of this drug effect continues to be debated, but etomidate does not carry the significant acute hemodynamic effects of other induction agents [22]. In critically ill patients, rather than using alternative induction agents, the use of etomidate may result in adrenal suppression. However, because the major cause of morbidity and mortality in septic patients is cardiovascular instability, the anesthesiologist's main focus should be on such matter. Merx and Weber [28] showed that the survival rate decreased in septic patients with cardiac dysfunction (septic cardiomyopathy). In conclusion, we feel that the net effect still favors the use of etomidate as an anesthetic induction agent.

\section{Miscellanies}

Thiopental is a derivative of barbituric acid, and is commonly used in the induction of anesthesia. It binds to gamma-aminobutyric acid (GABA) receptors, and is known to enhance GABA reactions associated with anesthetic potency. Thiopental also induce anti-inflammatory cytokines, such as interleukin-10 from LPS-stimulated mononuclear cells in vitro [29]. However, in guinea pig hearts, along with propofol and midazolam, thiopental decreased cardiac contractility, and it showed adverse effects potentiating LPS induced systemic hypotension [30,31]. Also, results showed greater depression of cardiac function in thiopental and propofol than ketamine or etomidate [11].

In the practice of anesthesia, midazolam is a commonly used benzodiazepine receptor agonist. In several studies, midazolam in vitro suppressed the activated macrophages, and inhibited the synthesis and release of interleukin-1, interleukin-6, and TNF- $\alpha$ [6]. Also, through the inhibition of NF-kB and p38 mitogen-activated protein kinase pathway midazolam inhibits LPS-stimulated inducible NO synthase, cyclooxygenase-2, and the expression of superoxide anion production [32]. Also, in patients with hypovolemia and heart disease, the use of other benzodiazepines with midazolam decreases systemic vascular resistance and blood pressure [33]. Propofol, together with midazolam, greatly decrease cardiac work in a dose-dependent fashion, showing the most adverse effects on cardiac stability [11]. Therefore, it is important to evaluate whether midazolam could cause hemodynamic instability and tissue perfusion impairment in septic patients or critical patients. Septic patients with mechanical ventilation are routinely administered with sedative and analgesic medication.

Recently, widely used alpha-2 agonist, such as dexmedetomidine, reduced vasopressor requirement and improved outcome in septic shock [34], as well as septic patients receiving dexmedetomidine had more days free of brain dysfunction (delirium and coma) and mechanical ventilation, and were less likely to die than those that received a lorazepam-based sedation regimen [35]. Also, dexmedetomidine reduced mortality rate and had an inhibitory effect on inflammatory response to endotoxin-induced shock in rats [36]. Although there are sedative agent in septic patients, dexmedetomidine may prove not to be a useful induction agent.

\section{Hemodynamic Stabilization and Support}

\section{Volume monitoring and fluid therapy}

Septic shock is primarily a form of distributional shock, characterizing in high cardiac output and low systemic vascular resistance. Hypotension may result due to decreased systemic vascular resistance in septic shock patients, and compromised tissue perfusion results in sequestration of blood in the microcirculation. Therefore, hemodynamic monitoring and support is important in maintaining an adequate perfusion of organs and cellular oxygen uptake in severe sepsis and septic 
shock patients. Impaired global perfusion occurs from not only ventricular dysfunction, but by arteriolar dilation, vascular obstruction, and volume depletion, which threatens the function of critical organs. Therefore, in treating septic patients, the anesthesiologist must pay close attention to the patients' intravascular volume status and cardiovascular function, and fluid resuscitation will be an important factor. Generally, CVP is probably the most used parameter for judging whether fluid should be administered.

Vincent and Weil [37] demonstrated that there is no evidence whether crystalloid or colloid is better than the other, and generally, the two are used in combination. It is not the type of the fluid, but the amount that is important. In order to restore the hemodynamic stability fluid, resuscitation is performed, according to the fluid challenge techniques. There are four important factors that must be kept in mind when performing the fluid challenge technique. There are (1) the type of fluid administered (e.g. crystalloid or colloid), (2) rate of fluid administration (e.g. 500-1,000 ml over 30 minutes), (3) the critical end points to be achieved (e.g. mean arterial pressure $>$ $70 \mathrm{mmHg}$, heart rate $<110$ beats/min), and (4) the safety limits (e.g. CVP $<15 \mathrm{mmHg}$ ). The SSC guideline for the management of severe sepsis and septic shock recommends that the initial hemodynamic resuscitation is to be done according to the protocol of early goal-directed therapy (EGDT) [38]. Following the SSC guideline (which propose a CVP target $>8 \mathrm{mmHg}$ for patients not receiving ventilation and $>12 \mathrm{mmHg}$ from patients receiving ventilation), this is evidence based on the fact that the level of adequate cardiac output is when filling pressures are $12-15 \mathrm{mmHg}$ [39].

There are also factors that must be kept in mind while maintaining anesthesia. First, there is a high possibility that most septic patients undergo fluid resuscitation in the ER or at the ward before a surgery. Secondly, these patients are generally spontaneously breathing, so CVP values tend to differ when mechanically ventilated during general anesthesia. Therefore, changes in CVP measurements can cause confusion during anesthesia management. Also, many studies report that fluid replacement, according to the patients filling pressure, lack in estimating the patients' intravascular volume [37,40]. In fact, it is dangerous to estimate the fluid responsiveness only through the preload measurement. When filling pressures are low, it is recommended that immediate fluid resuscitation must be initiated with careful monitoring, along with a fluid challenge test to a point which CVP increases at least $2 \mathrm{mmHg}$ [41]. When CVP and PAOP is below $8 \mathrm{mmHg}$ and $12 \mathrm{mmHg}$, respectively, it is difficult to directly reflect volume responsiveness. Therefore, excessive fluid resuscitation may lead to fluid overload. In result, the septic patient will develop pulmonary edema and progress to ARDS [42]. Also, in mechanically ventilated patients or those with increased intra-abdominal or intrathoracic pressure, if CVP is used as an indicator for fluid resuscitation, many times the patient will be under-resuscitation, resulting in organ dysfunction [43]. The main reason for the lack of correlation between the values of CVP and blood volume is that the body does everything possible to maintain homeostasis [44]. Therefore, precise and trustworthy methods of measurement to guide the fluid resuscitation are required. By using a transthoracic echocardiography, the ventricular filling, ventricular function, vasodilation, systolic failure, systolic and diastolic failure, or right ventricular failure can easily be evaluated $[45,46]$. Particularly In patients with septic shock, echocardiography can be used to guide fluid resuscitation by measuring the collapsibility of the inferior vena cava [47].

Dynamic indices derived from the arterial pressure waveform, which are affected by cyclic changes in pleural pressure during mechanical ventilation, such as stroke volume variation (SVV) and pulse pressure variation (PPV), have also been proven to be a more reliable indicators in response to volume challenge in conditions other than sepsis. SVV is measured for 30 s at a time, and it determines the variation of stroke volume in relation to the mean stroke volume. Normally, this measure should be below $10 \%$. SVV > 13\% may indicate hypovolemia. Marx et al. [48] demonstrated in a prospective study of patients with severe sepsis or septic shock that SVV might be superior to CVP and PAOP with respect to the estimation of cardiac preload and volume resuscitation effects. Furthermore, a threshold value for PPV of $13 \%$ (calculated as maximum pulse pressure minus minimum pulse pressure divided by the average and converted to percentage) was highly sensitive and specific in distinguishing the responders and nonresponders [49]. Moreover, because of the irregular filling times, in order to prevent inconsistent pulse pressure, cardiac rhythm must be regular.

During passive mechanical ventilation, inferior vena cava diameter tends to increase during inflation, and tends to decrease during expiration. In a mechanically ventilated septic patient, variations in vena cava diameter were fairly accurate in predicting the fluid responsiveness (eg, positive and negative predictive values of $93 \%$ and $92 \%$, respectively) [50]. Also, in a study subjected to patients with sepsis and acute lung injury, a superior vena cava collapsibility index $>36 \%$ predicted a significant, fluid-induced rise in cardiac output with a sensitivity of $90 \%$ and specificity of $100 \%$ [51]. Therefore, in predicting the fluid responsiveness in septic patients, dynamic values, such as SVV, PPV, peak aortic blood flow velocity variation, and respiratory variation in vena cava diameters can provide useful information.

The optimal transfusion triggers are different among the critically ill patients, but Rivers et al. suggested that in EGDT the goal for hematocrit should be $30 \%$ [38]. However, the SSC 
guidelines for the management of severe sepsis and septic shock recommends that outside the initial 6 hours period, after diagnosis of sepsis, red blood cell transfusion should be aimed at 7.0-9.0 g/dl, when hemoglobin falls below 7.0 $\mathrm{g} / \mathrm{dl}$ [52]. However, some suggest that transfusion thresholds should be reevaluated towards higher levels [53]. Currently, the multicenter European study is focusing on studying the blood transfusion strategies between maintaining hemoglobin concentrations of $7-9 \mathrm{~g} / \mathrm{dl}$ and above $9 \mathrm{~g} / \mathrm{dl}$. Until further data is revealed, careful transfusion of blood while evaluating the risks and benefits among each patient is needed.

\section{Vasopressor and inotropic therapy}

Vasopressor therapy is often needed in maintaining the perfusion and septic shock patients. It is even started early before the hypovolemia is completely corrected. It is still a disputed debate concerning which vasoactive agent is better than the other in severe sepsis and septic shock patients, and there seems to be no evidential congruity between the individuals. Current choice of vasoactive agents depends on the anesthesiologists' preference.

An arterial catheter is rapidly placed in order to assess the blood pressure in septic shock patients. Vasopressors are usually used when the mean arterial pressure falls below 65 $\mathrm{mmHg}$, with prior adequate fluid resuscitation or together with fluid resuscitation. Dopamine and norepinephrine are generally first-choice agents in treating septic shock. Epinephrine, phenylepherine, and vasopressin are not recommended as firstchoice agents in treating septic shock. However, in patients who are unresponsive to maximum doses of dopamine and norepinephrine with adequate fluid resuscitation, epinephrine can be used as a first alternative agent [52]. Also, in refractory shock patients who are unresponsive to adequate fluid resuscitation and high-dose conventional vasopressors can consider using low dose vasopressin, as a salvage therapy (infusion rates of $0.01-0.04 \mathrm{U} / \mathrm{min}$ ) [52]. Vasopressin barely has pressure effects in normal patients, but in septic shock patients with inadequate plasma vasopressin concentrations, low doses increase the mean arterial pressure, as well as decrease the need of other vasopressors [54]. The use of low-dose dopamine for renal protection is no longer recommended. Dobutamine is recommended as the inotropic agent of choice in increasing cardiac output in patients with sepsis-associated myocardial dysfunction, and is often used in combination with dopamine or norepinephrine. However, dobutamine must not be used in order to increase cardiac output above physiologic levels [55].

Although normalizing overall hemodynamic parameters does not suggest adequate tissue perfusion or oxygenation, vasoactive therapy must be continued based on the overall hemodynamic and oxygenation parameters, including mixed venous oxygenation saturation and blood lactate levels until a better method for estimating tissue perfusion and oxygenation turns up. Currently, there is no evidence on the superiority of one vasopressor against another in treating septic shock, but any one must be ready for use prior to induction of anesthesia.

\section{Conclusions}

Most often, septic patients portray instable hemodynamic states, due to hypotension or cardiomyopathy, caused by vasodilation, thus, impairing global tissue perfusion and oxygenation threatening functions of critical organs. Before induction, there are three things an an anesthesiologist must keep in mind. First, because arterial blood pressure, prior to surgery, does not represent adequate perfusion status of the patient, mental status, urine output and skin perfusion should always be evaluated. Anesthesiologist should evaluate the myocardial performance and hemodynamic monitoring for preload estimation of the patient by inserting a CVP catheter and evaluating the volume status. However, because it is difficult to accurately evaluate the intravascular volume only by CVP measurements, the additional use of transthoracic echocardiography, which the ventricular filling, ventricular function can be evaluated. Secondly, In order to hemodynamically stabilize septic patients, adequate fluid resuscitation or blood transfusion must be given before induction. However, if blood pressure is unstable, despite these efforts, vasopressors and inotropic drugs must be administered to be used so that the cardiovascular reserve can maximally be restored in these patients. Thirdly, the safest induction agents must be used for creating unconsciousness during induction. The ideal choice of medications for facilitating intubation in this setting is often a difficult one. The anesthesiologist must understand the acknowledged benefits and limitations of each drug in facilitating intubation in this population, especially on cardiovascular stability, onset, duration, and the effects on spontaneous ventilation.

Most intravenous anesthetics have anti-inflammatory effects so they respond well to septic patients. However, it may be useful to use drugs, such as ketamine or etomidate, which carry less cardiovascular instability effects than propofol, thiopental, and midazolam. In order to decrease ketamine induced emergence delirium, it may be useful to combine midazolam or propofol. Also, if reversal of etomidate induced adrenocortical suppression is necessary, the corticosteroid replacement therapy can be carried out.

\section{References}

1. Annane D, Aegerter P, Jars-Guincestre MC, Guidet B. Current epidemiology of septic shock: the CUB-Réa Network. Am J Respir 
Crit Care Med 2003; 168: 165-72.

2. Levy MM, Fink MP, Marshall JC, Abraham E, Angus D, Cook D, et al. 2001 SCCM/ESICM/ACCP/ATS/SIS International Sepsis Definitions Conference. Crit Care Med 2003; 31: 1250-6.

3. Basu S, Mutschler DK, Larsson AO, Kiiski R, Nordgren A, Eriksson MB. Propofol (Diprivan-EDTA) counteracts oxidative injury and deterioration of the arterial oxygen tension during experimental septic shock. Resuscitation 2001; 50: 341-8.

4. Taniguchi T, Yamamoto K, Ohmoto N, Ohta K, Kobayashi T. Effects of propofol on hemodynamic and inflammatory responses to endotoxemia in rats. Crit Care Med 2000; 28: 1101-6.

5. Mikawa K, Nishina K, Kodama S, Obara H. Propofol attenuates diaphragmatic dysfunction induced by septic peritonitis in hamsters. Anesthesiology 2001; 94: 652-60.

6. Inada T, Taniuchi S, Shingu K, Kobayashi Y, Fujisawa J, Nakao S. Propofol depressed neutrophil hydrogen peroxide production more than midazolam, whereas adhesion molecule expression was minimally affected by both anesthetics in rats with abdominal sepsis. Anesth Analg 2001; 92: 437-41.

7. Tang J, Sun Y, Wu WK, Zhong T, Liu Y, Xiao J, et al. Propofol lowers serum PF4 level and partially corrects hypercoagulopathy in endotoxemic rats. Biochim Biophys Acta 2010; 1804: 1895-901.

8. Taniguchi T, Kanakura H, Takemoto Y, Kidani Y, Yamamoto K. Effects of ketamine and propofol on the ratio of interleukin-6 to interleukin-10 during endotoxemia in rats. Tohoku J Exp Med 2003; 200: 85-92.

9. Reich DL, Hossain S, Krol M, Baez B, Patel P, Bernstein A, et al. Predictors of hypotension after induction of general anesthesia. Anesth Analg 2005; 101: 622-8.

10. Booke M, Armstrong C, Hinder F, Conroy B, Traber LD, Traber DL. The effects of propofol on hemodynamics and renal blood flow in healthy and in septic sheep, and combined with fentanyl in septic sheep. Anesth Analg 1996; 82: 738-43.

11. Zausig YA, Busse H, Lunz D, Sinner B, Zink W, Graf BM. Cardiac effects of induction agents in the septic rat heart. Crit Care 2009; 13: R144.

12. Larsen B, Hoff G, Wilhelm W, Buchinger H, Wanner GA, Bauer M. Effect of intravenous anesthetics on spontaneous and endotoxinstimulated cytokine response in cultured human whole blood. Anesthesiology 1998; 89: 1218-27.

13. Helmy SA, Al-Attiyah RJ. The immunomodulatory effects of prolonged intravenous infusion of propofol versus midazolam in critically ill surgical patients. Anaesthesia 2001; 56: 4-8.

14. Morris C, Perris A, Klein J, Mahoney P. Anaesthesia in haemodynamically compromised emergency patients: does ketamine represent the best choice of induction agent? Anaesthesia 2009; 64: 532-9.

15. Graf BM, Vicenzi MN, Martin E, Bosnjak ZJ, Stowe DF. Ketamine has stereospecific effects in the isolated perfused guinea pig heart. Anesthesiology 1995; 82: 1426-37.

16. Yu M, Shao D, Liu J, Zhu J, Zhang Z, Xu J. Effects of ketamine on levels of cytokines, NF-kappaB and TLRs in rat intestine during CLP-induced sepsis. Int Immunopharmacol 2007; 7: 1076-82.

17. Kawasaki T, Ogata M, Kawasaki C, Ogata J, Inoue Y, Shigematsu A. Ketamine suppresses proinflammatory cytokine production in human whole blood in vitro. Anesth Analg 1999; 89: 665-9.
18. Modig J. Positive effects of ketamine v. metomidate anesthesia on cardiovascular function, oxygen delivery and survival. Studies with a porcine endotoxin model. Acta Chir Scand 1987; 153: 7-13.

19. Taniguchi T, Shibata K, Yamamoto K. Ketamine inhibits endotoxininduced shock in rats. Anesthesiology 2001; 95: 928-32.

20. Busch CJ, Spohr FA, Motsch J, Gebhard MM, Martin EO, Weimann J. Effects of ketamine on hypoxic pulmonary vasoconstriction in the isolated perfused lungs of endotoxaemic mice. Eur J Anaesthesiol 2010; 27: 61-6.

21. Oglesby AJ. Should etomidate be the induction agent of choice for rapid sequence intubation in the emergency department? Emerg Med J 2004; 21: 655-9.

22. Bergen JM, Smith DC. A review of etomidate for rapid sequence intubation in the emergency department. J Emerg Med 1997; 15: 221-30.

23. Sprung CL, Annane D, Keh D, Moreno R, Singer M, Freivogel K, et al. Hydrocortisone therapy for patients with septic shock. N Engl J Med 2008; 358: 111-24.

24. Annane D, Sebille V, Charpentier C, Bollaert PE, Francois B, Korach JM, et al. Effect of treatment with low doses of hydrocortisone and fludrocortisone on mortality in patients with septic shock. JAMA 2002; 288: 862-71.

25. Kim TY, Rhee JE, Kim KS, Cha WC, Suh GJ, Jung SK. Etomidate should be used carefully for emergent endotracheal intubation in patients with septic shock. J Korean Med Sci 2008; 23: 988-91.

26. Murray H, Marik PE. Etomidate for endotracheal intubation in sepsis: acknowledging the good while accepting the bad. Chest 2005; 127: 707-9.

27. Arbous MS, Grobbee DE, van Kleef JW, de Lange JJ, Spoormans HH, Touw P, et al. Mortality associated with anaesthesia: a qualitative analysis to identify risk factors. Anaesthesia 2001; 56: 1141-53.

28. Merx MW, Weber C. Sepsis and the heart. Circulation 2007; 116: 793-802.

29. Takaono M, Yogosawa T, Okawa-Takatsuji M, Aotsuka S. Effects of intravenous anesthetics on interleukin (IL)-6 and IL-10 production by lipopolysaccharide-stimulated mononuclear cells from healthy volunteers. Acta Anaesthesiol Scand 2002; 46: 176-9.

30. Kao SJ, Su CF, Liu DD, Chen HI. Endotoxin-induced acute lung injury and organ dysfunction are attenuated by pentobarbital anaesthesia. Clin Exp Pharmacol Physiol 2007; 34: 480-7.

31. Stowe DF, Bosnjak ZJ, Kampine JP. Comparison of etomidate, ketamine, midazolam, propofol, and thiopental on function and metabolism of isolated hearts. Anesth Analg1992; 74: 547-58.

32. Kim SN, Son SC, Lee SM, Kim CS, Yoo DG, Lee SK, et al. Midazolam inhibits proinflammatory mediators in the lipopolysaccharideactivated macrophage. Anesthesiology 2006; 105: 105-10.

33. Shafer A. Complications of sedation with midazolam in the intensive care unit and a comparison with other sedative regimens. Crit Care Med 1998; 26: 947-56.

34. Pichot C, Geloen A, Ghignone M, Quintin L. Alpha-2 agonists to reduce vasopressor requirements in septic shock? Med Hypotheses 2010; 75: 652-6.

35. Pandharipande PP, Sanders RD, Girard TD, McGrane S, Thompson JL, Shintani AK, et al. Effect of dexmedetomidine versus lorazepam on outcome in patients with sepsis: an a priori-designed analysis of the MENDS randomized controlled trial. Crit Care 2010; 14: R38. 
36. Taniguchi T, Kidani Y, Kanakura H, Takemoto Y, Yamamoto K. Effects of dexmedetomidine on mortality rate and inflammatory responses to endotoxin-induced shock in rats. Crit Care Med 2004; 32: $1322-6$.

37. Vincent JL, Weil MH. Fluid challenge revisited. Crit Care Med 2006; 34: 1333-7.

38. Rivers E, Nguyen B, Havstad S, Ressler J, Muzzin A, Knoblich B, et al. Early goal-directed therapy in the treatment of severe sepsis and septic shock. N Engl J Med 2001; 345: 1368-77.

39. Hollenberg SM, Ahrens TS, Annane D, Astiz ME, Chalfin DB, Dasta JF, et al. Practice parameters for hemodynamic support of sepsis in adult patients: 2004 update. Crit Care Med 2004; 32: 1928-48.

40. Michard F, Teboul JL. Predicting fluid responsiveness in ICU patients: a critical analysis of the evidence. Chest 2002; 121: 2000-8.

41. Antonelli M, Levy M, Andrews PJ, Chastre J, Hudson LD, Manthous C, et al. Hemodynamic monitoring in shock and implications for management. International Consensus Conference, Paris, France, 27-28 April 2006. Intensive Care Med 2007; 33: 575-90.

42. Osman D, Ridel C, Ray P, Monnet X, Anguel N, Richard C, et al. Cardiac filling pressures are not appropriate to predict hemodynamic response to volume challenge. Crit Care Med 2007; 35: 64-8.

43. Cheatham ML. It is time to pay attention--now more than ever! Crit Care Med 2007; 35: 1629-30.

44. Gelman S. Venous function and central venous pressure: a physiologic story. Anesthesiology 2008; 108: 735-48.

45. Faris JG, Veltman MG, Royse CF. Limited transthoracic echocardiography assessment in anaesthesia and critical care. Best Pract Res Clin Anaesthesiol 2009; 23: 285-98.

46. Royse CF, Seah JL, Donelan L, Royse AG. Point of care ultrasound for basic haemodynamic assessment: novice compared with an expert operator. Anaesthesia 2006; 61: 849-55.

47. Griffee MJ, Merkel MJ, Wei KS. The role of echocardiography in hemodynamic assessment of septic shock. Crit Care Clin 2010; 26 : 365-82.

48. Marx G, Cope T, McCrossan L, Swaraj S, Cowan C, Mostafa SM, et al. Assessing fluid responsiveness by stroke volume variation in mechanically ventilated patients with severe sepsis. Eur J Anaesthesiol 2004; 21: 132-8.

49. Michard F, Boussat S, Chemla D, Anguel N, Mercat A, Lecarpentier Y, et al. Relation between respiratory changes in arterial pulse pressure and fluid responsiveness in septic patients with acute circulatory failure. Am J Respir Crit Care Med 2000; 162: 134-8.

50. Feissel M, Michard F, Faller JP, Teboul JL. The respiratory variation in inferior vena cava diameter as a guide to fluid therapy. Intensive Care Med 2004; 30: 1834-7.

51. Vieillard-Baron A, Chergui K, Rabiller A, Peyrouset O, Page B, Beauchet A, et al. Superior vena caval collapsibility as a gauge of volume status in ventilated septic patients. Intensive Care Med 2004; 30: 1734-9.

52. Dellinger RP, Levy MM, Carlet JM, Bion J, Parker MM, Jaeschke $\mathrm{R}$, et al. Surviving Sepsis Campaign: international guidelines for management of severe sepsis and septic shock: 2008. Crit Care Med 2008; 36: 296-327.

53. Leone M, Bourgoin A, Cambon S, Dubuc M, Albanese J, Martin C. Empirical antimicrobial therapy of septic shock patients: adequacy and impact on the outcome. Crit Care Med 2003; 31: 462-7.

54. Tsuneyoshi I, Yamada H, Kakihana Y, Nakamura M, Nakano Y, Boyle WA 3rd. Hemodynamic and metabolic effects of low-dose vasopressin infusions in vasodilatory septic shock. Crit Care Med 2001; 29: 487-93.

55. Beale RJ, Hollenberg SM, Vincent JL, Parrillo JE. Vasopressor and inotropic support in septic shock: an evidence-based review. Crit Care Med 2004; 32(11 Suppl): S455-65. 